\title{
Implications of Functionally Different Synaptic \\ Inputs for Neuronal Gain and Computational \\ Properties of Fly Visual Interneurons
}

Jan Grewe, Nélia Matos, Martin Egelhaaf and Anne-Kathrin Warzecha

J Neurophysiol 96:1838-1847, 2006. First published Jun 21, 2006; doi:10.1152/jn.00170.2006

You might find this additional information useful...

This article cites 51 articles, 22 of which you can access free at:

http://jn.physiology.org/cgi/content/full/96/4/1838\#BIBL

Updated information and services including high-resolution figures, can be found at:

http://jn.physiology.org/cgi/content/full/96/4/1838

Additional material and information about Journal of Neurophysiology can be found at:

http://www.the-aps.org/publications/jn

This information is current as of September 14, 2006 . 


\title{
Implications of Functionally Different Synaptic Inputs for Neuronal Gain and Computational Properties of Fly Visual Interneurons
}

\author{
Jan Grewe, ${ }^{1,2}$ Nélia Matos, ${ }^{1}$ Martin Egelhaaf, ${ }^{1}$ and Anne-Kathrin Warzecha ${ }^{2,1}$ \\ ${ }^{1}$ Lehrstuhl für Neurobiologie, Universität Bielefeld, Bielefeld; and ${ }^{2}$ Psychologisches Institut II, Westfälische Wilhelms-Universität Münster, \\ Münster, Germany
}

Submitted 16 February 2006; accepted in final form 16 June 2006

\begin{abstract}
Grewe, Jan, Nélia Matos, Martin Egelhaaf, and Anne-Kathrin Warzecha. Implications of functionally different synaptic inputs for neuronal gain and computational properties of fly visual interneurons. J Neurophysiol 96: 1838-1847, 2006. First published June 21, 2006; doi:10.1152/jn.00170.2006. Neurons embedded in networks are thought to receive synaptic inputs that do not drive them on their own, but modulate the responsiveness to driving input. Although studies on brain slices have led to detailed knowledge of how nondriving input affects dendritic integration, its origin and functional implications remain unclear. We tackle this issue using an ensemble of fly widefield visual interneurons. These neurons offer the opportunity not only to combine in vivo recording techniques and natural sensory stimulation but also to interpret electrophysiological results in a behavioral context. By targeted manipulation of the animal's visual input we find a pronounced modulating impact of nondriving input, whereas functionally important cellular properties like direction tuning and the coding of pattern velocity are left almost unaffected. We propose that the integration of functionally different synaptic inputs is a mechanism that immanently equalizes the ensemble's sensitivity irrespective of the specific stimulus conditions.
\end{abstract}

\section{N T R O D U C T I O N}

Dendritic integration is a central and often highly nonlinear stage of neuronal information processing. The many different presynaptic signals a neuron receives have been classified as drivers and modulators (Sherman and Guillery 1998). Driving input is thought to carry the relevant information, whereas the modulating input shapes the neuronal computations without any driving contribution. Shunting inhibition is one example of modulating input, which may even veto the driving input (Koch et al. 1983). Cortical neurons are assumed to be continually bombarded with excitatory and inhibitory inputs induced by background network activity. The impact of background activity leads, e.g., to a drop in input resistances in vivo as compared with those in vitro or in anesthetized animals when there is only reduced network activity (Destexhe et al. 2003). Accordingly, the background activity has been proposed to be a mechanism that modulates the gain of a neuron (Chance et al. 2002; Mitchell and Silver 2003; Prescott and De Koninck 2003). Despite great methodological advances such as the dynamic-clamp technique, applied to mimic background activity in slice preparations (Prinz et al. 2004), conclusions about the functional significance and origin of background input are still limited.

Here we address the functional significance of modulating synaptic background input linking cellular physiology with the

Address for reprint requests and other correspondence: J. Grewe, Lehrstuhl für Neurobiologie, Universität Bielefeld, Postfach 1001 31, 33501 Bielefeld, Germany (E-mail: jan.grewe@uni-bielefeld.de). functional aspects of encoding and representing visual stimuli. We investigate how visual shunting input affects the encoding of visual motion stimuli in fly visual interneurons. The task of these tangential cells (TCs) is to evaluate optic flow patterns, i.e., the retinal image flow evoked during self-motion of the animal (Borst and Haag 2002; Egelhaaf et al. 2002, 2005; Hausen 1984). Optic flow patterns are the only source of information about the environmental layout when airborne. TCs spatially integrate the excitatory and inhibitory outputs of thousands of retinotopically arranged local motion-sensitive elements (Brotz et al. 1996). Accordingly, TCs have receptive fields that cover large parts of the visual field and are tuned to optic flow as is generated on the eyes during certain types of translational or rotational self-motion of the animal (Egelhaaf et al. 2002; Kern et al. 2005; Krapp et al. 2001; van Hateren et al. 2005). The activation ratio of excitatory and inhibitory inputs depends on stimulus parameters as pattern contrast and texture or the direction and velocity of visual motion. Even during motion in the preferred direction, which distinctly depolarizes the cell, the inhibitory inputs are activated, although to a much smaller extent than the excitatory input (Borst et al. 1995; Single et al. 1997). Motion orthogonal to the preferred direction is assumed to activate both types of inputs in approximately equal shares, whereas during null-direction motion the inhibitory input predominates. Fly TCs offer the opportunity to investigate under in vivo conditions the influence of selectively manipulated sensory input in a well-established functional context. In this study we examined the functional consequences of modulating input on neuronal sensitivity, direction tuning, and the representation of the time course of pattern velocity. Coherent motion as driving input and balanced motion noise as the modulating background input were combined for visual stimulation. This decomposition of the visual input into a driving and a modulating component was done for the sake of systems analysis only, although under natural conditions TCs are exposed to optic flow containing motion vectors in a wide range of directions driving its activity to a different extent or not at all. Thus in behavioral situations TCs receive inputs that have both driving and modulating influences.

\section{METHODS}

\section{Electrophysiology}

Experiments were carried out on 1- to 7-day-old female blowflies (Calliphora vicina). The animals were prepared as described in

The costs of publication of this article were defrayed in part by the payment of page charges. The article must therefore be hereby marked "advertisement" in accordance with 18 U.S.C. Section 1734 solely to indicate this fact. 
Warzecha et al. (1993). In short: the flies were briefly anesthetized with $\mathrm{CO}_{2}$ and fixed ventral side up on a small piece of glass. The head was bent down and waxed to the thorax to allow access to the backside of the head. The head-capsule was opened and tissues such as air sacs, fat bodies, and trachea that cover the lobula plate were removed. In some preparations we also removed the gut to eliminate peristaltic movements that can cause disturbances. After preparation, the flies were adjusted according to the symmetry of the deep pseudopupil (Franceschini 1975) to ensure that different animals received the same visual input.

\section{Extracellular recordings}

The H1 neuron was recorded extracellulary with self-made tungsten electrodes. A Ringer-filled glass capillary, through which we could provide Ringer solution if necessary (for composition see Hausen 1982a), was used as an indifferent electrode. The recorded signals were amplified by a factor of 3,000, band-pass filtered (corner frequencies of $300 \mathrm{~Hz}$ and $3 \mathrm{kHz}$ ), and subsequently passed through a threshold device that transforms detected spikes into uniform pulses.

The H1 neuron was recorded in the left half of the brain in its output arborization. It can be easily identified by its preferred direction, i.e., back-to-front motion in front of the right eye.

\section{Intracellular recordings}

VS (vertical system) neurons were recorded in or close to their main dendrite with sharp electrodes pulled on a Brown/Flaming P-97 Puller (Sutter Instruments) and filled with $2 \mathrm{M} \mathrm{KCl}$. The electrodes had resistances between 30 and $40 \mathrm{M} \Omega$ and the electrode holder's silver wire was chlorided before every recording. We used a SEC 10-L amplifier (npi electronics, Tamm, Germany) operated in bridge or discontinuous current-clamp (DCC) mode. In DCC mode the switching frequency was about $8 \mathrm{kHz}$.

The raw data traces of intra- and extracellular recordings as well as the thresholded $\mathrm{H} 1$ spike trains were sampled at $4 \mathrm{kHz}$ (DaqBoard2000; IOtech, Cleveland, $\mathrm{OH}$ ) and stored on hard disk for off-line analysis. The program for data acquisition was written in Delphi 7 (Borland Software).

\section{Visual stimulation}

Visual stimuli were generated using a VSG 2/3 graphics card (Cambridge Research Systems, Cambridge, UK) and presented on a Joyce Scope DM5 monochrome (P-31 phosphor) monitor (Joyce Electronics, Cambridge, UK). As seen by the fly, the monitor screen $(464 \times 375$ pixel spatial resolution) had a horizontal and vertical extent of 127 and $120^{\circ}$, respectively. The screen center was positioned at $0^{\circ}$ elevation and $20^{\circ}$ azimuth. At this point, one pixel had a vertical and horizontal extent of about $0.5^{\circ}$.

Whereas the frame rate was $300 \mathrm{~Hz}$, new stimulus pictures were presented with $150 \mathrm{~Hz}$. We could disprove time-locking of H1 spikes to either frequency by cross-correlating 200 response traces to identical stimulation (data not shown).

The stimuli consisted of two sets of dots moving on the black screen (luminance: $0.001 \mathrm{~cd} / \mathrm{m}^{2}$ ). The first set of dots, the motion dots, were randomly positioned in the first frame and moved coherently in the preferred direction of the recorded TC. These served as driving input, forming the sensory signal to be encoded by the TCs. Each dot had a vertical and horizontal extent of three pixels, resulting in an angular size of about $1.5 \times 1.5^{\circ}$ as seen by the fly; it was surrounded by a $6^{\circ}$ "forbidden zone" in which no other dot was allowed to be placed. By varying the number of dots (between 4 and 64) different movement strengths were produced. The motion dots were always presented at full brightness $\left(300 \mathrm{~cd} / \mathrm{m}^{2}\right)$. The second set of dots consisted of 112 noise dots, which have the same size and forbidden zone as those of the motion dots. The noise dots were designed to constitute the modulating background input. Initially the noise dots were also randomly placed. In subsequent frames they performed a random walk, in which eight directions (the horizontal, vertical, and diagonal directions) were possible. For each time step the direction of each dot was chosen randomly from a predefined distribution. This distribution of directions was defined so that the directions into which the noise dots moved were balanced, i.e., the resulting motion noise did not, on average, excite or inhibit the recorded neuron. Balancing was done in control experiments for one sample VS cell and H1 cell. The distribution of movement directions that led to the best balancing, that is, with no clear excitation or inhibition, was used for all other experiments on the same cell type. The strength of the motion noise was altered by changing the brightness of the noise dots to about 30 $\mathrm{cd} / \mathrm{m}^{2}$ (weak motion noise), $150 \mathrm{~cd} / \mathrm{m}^{2}$ (medium motion noise), and $300 \mathrm{~cd} / \mathrm{m}^{2}$ (strong motion noise). Even the lowest brightness value was sufficient to significantly affect the neuronal responses, increasing the firing rate of the $\mathrm{H} 1$ neuron when dots of this brightness moved in the cell's preferred direction.

As mentioned above, the dots were surrounded by a "forbidden zone," in which no other dot occurred. This zone was introduced to prevent the motion dots and noise dots from interacting on a local basis, i.e., generating apparent motion. In flies, adapted to total darkness, it is possible to evoke a weak direction-selective response in H1 when the two stimuli of an apparent motion paradigm are separated $\leq 12^{\circ}$ (Schuling et al. 1989). Although the flies in our experiments were not completely dark adapted, we could show in a control experiment that increasing the forbidden zone to $>12^{\circ}$ (with a reduced number of dots) or completely separating motion and noise dots (coherent motion in the top third of the screen and motion noise in the bottom third or vice versa) does not significantly alter the effect of motion noise on $\mathrm{H} 1$ responses.

\section{Measurements of TC input resistance}

The input resistances of VS cells were measured by injecting 200-ms pulses of constant hyperpolarizing current $(-0.75$ to -1.5 $\mathrm{nA}$ ) into the cells. Trials with and without current were recorded in a pseudorandom order that allowed calculation of the input resistances from direct comparisons of the corresponding response sections (Fig. $2 A)$.

\section{Gain modulation by visual motion noise}

To investigate the consequences of motion noise on the sensitivity of TCs for preferred direction motion, we tested the neuronal responses to motion stimuli of five different strengths, determined by the number of dots moving on the screen $(4,8,16,32$, and 64 dots) combined with (or without) motion noise. As mentioned above, three different strengths of motion noise were used. Each trial consisted of three test sections: 1) coherent motion with the actual number of motion dots moving, 2) the motion noise alone at a given intensity, and 3) coherent motion and motion noise combined. These were alternated with so-called reference sections in which the maximum number of motion dots moved and breaks showing a stationary image (Table 1). This image was the first image of the following section.

\section{Direction tuning in the presence of motion noise}

A similar stimulus protocol was used to test the direction selectivity of TCs (Table 1, right). In these reference sections the maximum number of motion dots moved in the preferred direction of the cell. In the test sections the coherently moving dots (16 or 64) were moved in one of the eight tested directions $\left(0-315^{\circ}\right.$ in $45^{\circ}$ steps $)$. To reduce the number of conditions (to have a reasonable number of trials for each condition) we used only the medium and strong motion noises. 
TABLE 1. Experimental protocol describing the image shown

\begin{tabular}{|c|c|c|}
\hline $\begin{array}{l}\text { Duration, } \\
\mathrm{s}\end{array}$ & Gain Modulation & Direction Tuning \\
\hline 0.5 & Blank screen & Blank screen \\
\hline \multicolumn{3}{|c|}{ Reference section } \\
\hline 1.0 & Pure motion & Pure motion \\
\hline 1.0 & Stationary image & Stationary image \\
\hline \multicolumn{3}{|c|}{ Test section I } \\
\hline 1.0 & Pure motion (4-64 dots) & Pure motion (various directions) \\
\hline 1.0 & Stationary image & Stationary image \\
\hline \multicolumn{3}{|c|}{ Reference section } \\
\hline 1.0 & Pure motion & Pure motion \\
\hline 1.0 & Stationary image & Stationary image \\
\hline \multicolumn{3}{|c|}{ Test section II } \\
\hline 1.0 & Noise (weak, medium, strong) & Noise (weak, strong) \\
\hline 1.0 & Stationary image & Stationary image \\
\hline \multicolumn{3}{|c|}{ Reference section } \\
\hline 1.0 & Pure motion & Pure motion \\
\hline 1.0 & Stationary image & Stationary image \\
\hline \multicolumn{3}{|c|}{ Test section III } \\
\hline 1.0 & Motion (4-64 dots) + noise & $\begin{array}{l}\text { Motion (various directions) }+ \\
\text { noise }\end{array}$ \\
\hline
\end{tabular}

\section{Representation of pattern velocity in the presence of motion noise}

We investigated the representation of pattern velocity with extracellular recordings of the $\mathrm{H} 1$ cell because this analysis requires stable recordings over a longer time than can routinely be accomplished by intracellular recording. The motion dot velocity was dynamically modulated (Fig. 6, left column). The underlying velocity profile was built up from sine waves of $1,3,5,7$, and $9 \mathrm{~Hz}$ at random phases. The frequencies were matched to the frequency band in which the $\mathrm{H} 1$ cell codes pattern velocity with a high gain (Warzecha et al. 1998). Here we used a stimulus protocol consisting of three test sections (dynamic motion alone, motion noise alone, and the combination of both, each of 1,000-ms duration). The test sections were separated by stationary image sections of 5,000-ms duration. The interstimulus interval between consecutive trials of any experiment was 5,000 ms. This time was used to save the data and to provide Ringer solution if necessary.

\section{Data analysis}

Off-line analysis was done with Matlab Release 14 (The MathWorks, Natick, MA). To analyze the performance of H1 to encode stimulus velocity (see above) we used the coherence analysis (Haag and Borst 1998; van Hateren and Snippe 2001) using the coherence function distributed with Matlab. For this purpose the responses were aligned with the velocity profile by removing the response delay calculated from the cross-correlation of the mean response and the velocity profile. Both stimulus and response vectors were zero padded to give 4,096 sample points. Because of the rectification nonlinearity inherent in the spike-generation process, the $\mathrm{H} 1$ can code pattern velocity only in its preferred direction. Therefore we rectified the stimulus velocity accordingly and set it to zero if the dots moved in the null direction. The coherence between stimulus and response was calculated using a 4,096-point Hanning window and an overlap of 2,048 points. The coherence analysis quantifies the similarity between the stimulus and the stimulus reconstructed from the responses using the best linear filter. In the case of a noise-free linear encoder the coherence is 1 for all frequencies. Deviations from unity could be a consequence of noise and of nonlinearities in the system. To separate these two effects the expected coherence (van Hateren and Snippe 2001) was used. The expected coherence is defined as the coherence between the individual responses and the "noise-free" average response. The deviation of the expected coherence from unity can be traced back to noise in the system. The difference between coherence and expected coherence arises from the nonlinearities.

To estimate the information that is transmitted by the neuronal responses about the stimulus the coherence rate was calculated from the coherence spectrum according to van Hateren and Snippe (2001)

$$
R_{\text {coh }}=-\int_{0}^{\infty} \log _{2}\left(1-\gamma^{2}\right) \mathrm{d} f
$$

where $R_{\text {coh }}$ is the coherence rate and $\gamma$ is the coherence. The expected coherence rate $\left(R_{\exp }\right)$ was calculated accordingly.

Because the interpretation of the coherence rate in terms of transmitted information depends on assumptions that neuronal noise is Gaussian and additive, an assumption that is not strictly correct, we additionally calculated the information rate with the measure developed by Brenner et al. (2000)

$$
I=\frac{1}{T} \int_{0}^{\tau} \mathrm{d} t\left[\frac{r(t)}{\bar{r}}\right] \log _{2}\left[\frac{r(t)}{\bar{r}}\right]
$$

where $T$ is the number of time bins, $r(t)$ is the time-dependent spike rate, and $\bar{r}$ is the average spike rate. Both measures implicitly assume that information is encoded by the spike rate of the neuron. This assumption appears justified as a first approximation because TC spikes were concluded to lock precisely to the stimulus in a frequency range of velocity fluctuations that are only weakly represented in TC responses (Kretzberg et al. 2001; Warzecha et al. 1998).

\section{RES U L T S}

We analyzed the nonlinear dendritic integration of driving and modulating synaptic input in two types of directionally selective, motion-sensitive neurons. 1) The $\mathrm{H} 1$ neuron (Hausen 1976) offers the opportunity of stable long-lasting extracellular recordings, which is required for a detailed analysis of coding properties (Bialek et al. 1991; Warzecha et al. 2000). The H1 cell is excited by back-to-front motion and inhibited by motion in the opposite direction within almost the entire visual field of one eye. It uses spikes to transmit information from one brain hemisphere to the other. 2) The VS cells (Hengstenberg 1982; Krapp et al. 1998) can be recorded intracellularly and respond to both downward and upward motion within a broad vertical stripe of the visual field with graded de- and hyperpolarizations, respectively. These graded membrane potential shifts are assumed to represent the summated postsynaptic potentials of the cell and can be recorded even close to the output terminal of the cells (e.g., Egelhaaf and Warzecha 1999). Because VS-graded depolarization may be superposed by spikes, reminiscent of the variable spikes observed in cortical neurons (e.g., Azouz and Gray 1999), VS cells can also be used to study the transformation of postsynaptic potentials into spikes. In this study we concentrate on two types of the $10 \mathrm{VS}$-cells, i.e., VS1 and VS2/3. Because VS2 and VS3 are two cells with largely overlapping receptive fields and virtually indistinguishable functional properties (Krapp et al. 1998), they are lumped here into one category of cells "VS2/3". The analyzed VS cells have their receptive fields in the frontal part of the visual field.

To test the influence of modulating input on the response to a driving visual signal, two types of stimuli were used (Fig. 1, 
insets). 1) The driving visual input consists of a variable number of dots moving coherently either in the cell's preferred or null direction. 2) The modulating input consists of dots moving in a variety of directions so that excitatory and inhibitory effects cancel each other ("motion noise"). The driving input and the motion noise can be presented individually or combined. The motion noise component then acts as a modulating background input (see METHODs for details).

\section{Balanced motion noise has shunting characteristics}

The motion noise was balanced having no pronounced driving effect on the membrane potential on its own (Fig. 1A, right shaded response section). To achieve balance, more dots were required with a motion component in the null direction than into the preferred direction of the respective cell. This is most likely a result of the stronger driving force for the excitatory currents than that for the inhibitory ones. Although the motion noise neither excites nor inhibits the neuron considerably, it increases the membrane potential fluctuations around the resting potential (Fig. 1A, right shaded area). These fluctuations were smaller in some recordings, like the one shown in Fig. 2. This might be a consequence of the slight hyperpolarizing effect of the motion noise in this experiment or of the different recording sites (close to the dendrites and close to the output terminals for the experiments shown in Figs. 1 and 2 , respectively).

To determine whether motion noise has shunting characteristics, we measured the changes in input resistance of VS neurons relative to the resting input resistance induced by motion noise stimulation and by null-direction motion stimulation, respectively (Fig. 1). The input resistance was estimated by injecting hyperpolarizing current pulses into the cells. The VS cell input resistances at rest were on average $6.81 \pm 2.56$
$\mathrm{M} \Omega$ ( $n=4$ cells), which is in the range reported previously (Borst and Haag 1996). However, the resting input resistances varied considerably. In one VS cell (shown in Fig. 1, $A$ and $B$ ) an input resistance of $10.5 \mathrm{M} \Omega$ at rest was measured, deviating much from the average. We did not find any obvious correlation between the difference in input resistance and the visually induced responses of VS cells. Anyway, during coherent nulldirection motion, when the cell is hyperpolarized, the input resistance is decreased relative to the resting input resistance $[4.71 \pm 2.05 \mathrm{M} \Omega ;-32.23 \pm 5.86 \%$ (Fig. $1, B$ and $C$ )]. Motion noise does not significantly depolarize or hyperpolarize the cell but it reduces the input resistance to $5.06 \pm 1.95 \mathrm{M} \Omega$ $[-26.05 \pm 6.52 \%$ (Fig. $1, B$ and $C$ )]. This reduction is in the same range, although slightly smaller, as the reduction of the input resistance during coherent null-direction motion. From these experiments we conclude that visual motion noise can be considered a shunting input comparable to the background input artificially induced in slice preparations (Chance et al. 2002; Mitchell and Silver 2003; Prescott and De Koninck 2003).

\section{Nonlinear integration of motion information}

To analyze the effect of motion noise on the neuronal gain we determined the response amplitudes of VS and $\mathrm{H} 1$ cells as a function of stimulus strength in the presence of motion noise of different levels. Stimulus strength was modified by varying the number of coherently moving dots. The strength of the motion noise was altered by increasing the brightness of the dots constituting the motion noise.

Whereas both graded postsynaptic potentials and spikes can be recorded in VS cells (Fig. 2A), the $\mathrm{H} 1$ cell allows only spike responses to be recorded, although for extended periods of time (Fig. 3). For both response modes of VS cells the cell's

A

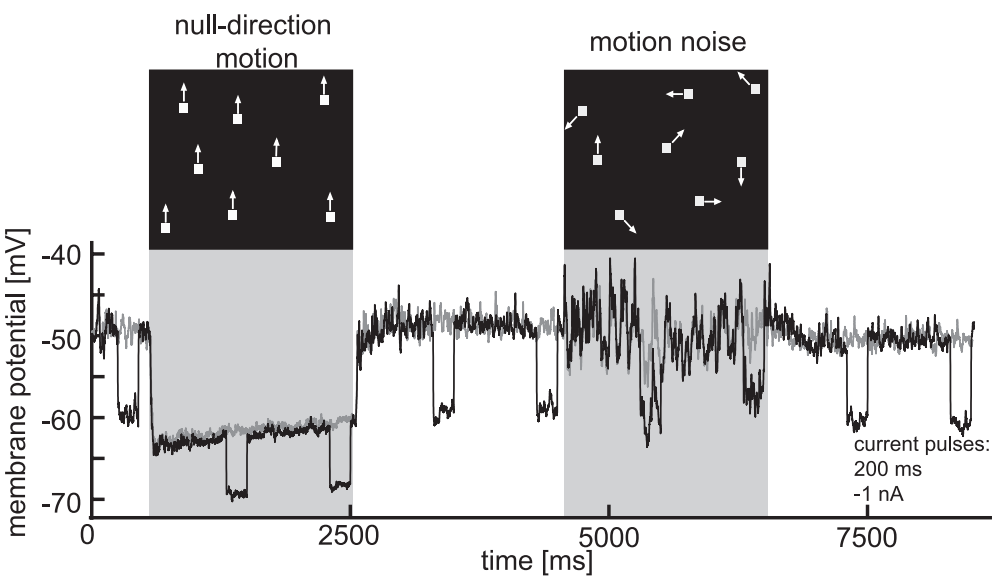

B

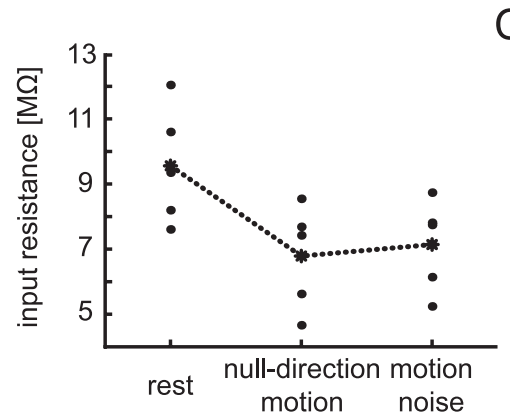

C

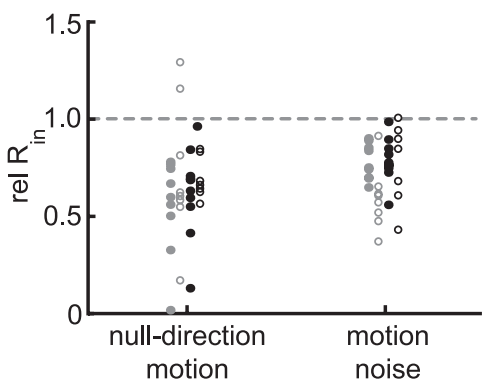

FIG. 1. Measurement of the tangential cell (TC) input resistance. $A$ : average response of one VS cell (10 trials) to coherent null-direction motion and to motion noise (first and second shaded areas). Strongest motion noise was chosen for this measurement. In half of the trials (black trace) 1-nA hyperpolarizing current pulses of 200-ms duration were injected into the cell. Input resistance was calculated from the difference between the "current responses" and the "no-current" responses (black and gray traces, which were recorded alternately). B: input resistance on single-trial basis (black dots) and on average across the 5 trials on this condition (asterisks) of the same cell as shown in $A$. Input resistances were measured at rest, during null-direction motion, and motion noise. $C$ : pooled results from 4 VS cells recorded in 4 different animals; each dot is the input resistance estimated in a single trial. Input resistances are normalized to the respective input resistances at rest. Different symbols denote results from the different cells. 

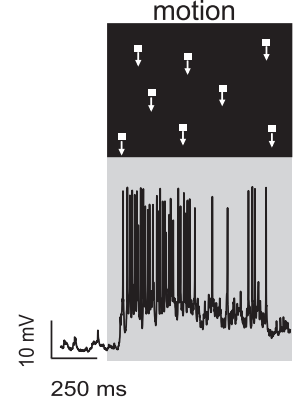

B
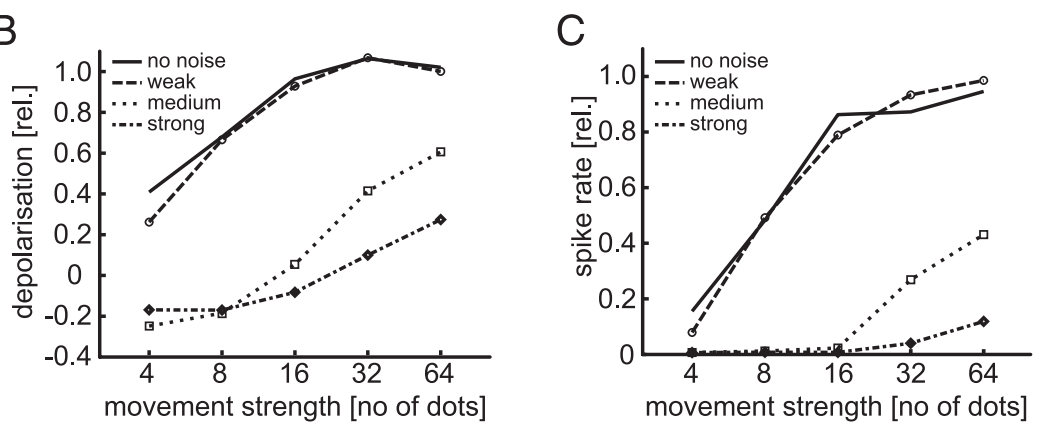

FIG. 2. Influence of motion noise on VS sensitivity to preferred direction motion. $A$ : individual VS1 responses to coherent preferred direction motion, motion noise, and the combination of coherent motion and motion noise recorded close to the axon terminal. These responses were evoked by the strongest motion stimulus (64 dots moving coherently) and the strongest motion noise (maximal brightness of the 112 motion noise dots). VS cells show graded shifts of the membrane potential superimposed with spikes. $B$ : input-output characteristics of the graded membrane potential shift on different motion noise intensities. Motion strength was varied by increasing the number of dots moving coherently, whereas the intensity of the motion noise was altered by varying the brightness of the dots constituting the motion noise (solid line, dashed line with open circles, dotted line with open squares, and dash-dotted line with open diamonds represent no, weak, medium, and strong motion noise, respectively). Response amplitudes are normalized to the response to the strongest coherent motion (64 dots moving in preferred direction). $C$ : same as $B$ but for the spike response of this cell. sensitivity to motion stimuli is reduced by motion noise, independent of motion strength (Figs. 2 and 3). Stronger motion noise produces larger reductions in response amplitude. This reduction cannot be explained by a summation of the respective responses to coherent motion and motion noise (Fig. $2 A$, left and middle sections). Thus the reduction in responsiveness is mainly a result of the shunting effect of the motion noise. This is true despite the slight hyperpolarization of about $-3.5 \mathrm{mV}$ for the strongest motion noise in the example shown in Fig. $2 A$ (middle column). Similar reductions were observed in other recordings in which motion noise led to slight depolarizations. The reduction in sensitivity for the driving input induced by coherent motion is similar for both the graded membrane potential shift and the spiking response of the VS recording (compare Fig. 2, $A$ and $B$ ). However, the threshold nonlinearity of spike generation is apparent because strong motion noise suppresses completely the spike responses to small and moderate motion strengths. Similar results were found in two other VS recordings and two recordings from other TCs (one HSE and one $\mathrm{CH}$ cell).

$\mathrm{H} 1$ is a spiking tangential cell that we recorded extracellularly. The responses of one sample recording are shown as peristimulus time histograms (PSTHs) in Fig. $3 A$. Figure $3 B$ shows the average characteristics measured in four cells (similar results were found in four additional recordings that are not included because a slightly different stimulus protocol was applied). As for VS cells $\mathrm{H} 1$ spike frequency increases with increasing stimulus strength. When the motion stimulus is combined with motion noise the response amplitude is reduced in a similar way as that found in VS cells. With respect to VS cells, this reduction is present even if the motion noise induces a slight increase of the spike rate on its own. Thus the interaction of the input mediated by coherent motion, on the one hand, and the input by motion noise, on the other hand, is far from linear. Shunting inhibition resulting from motion noise has severe conse-
A

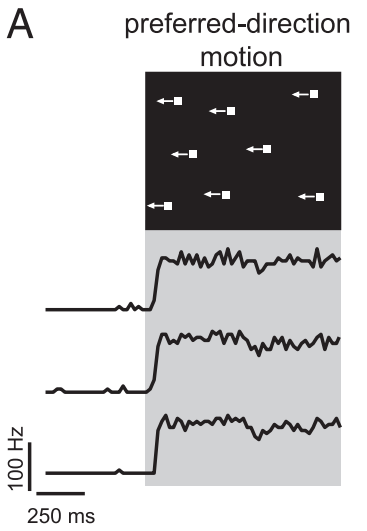

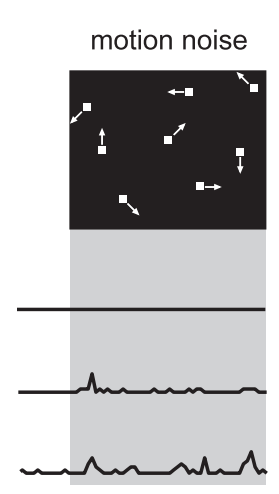

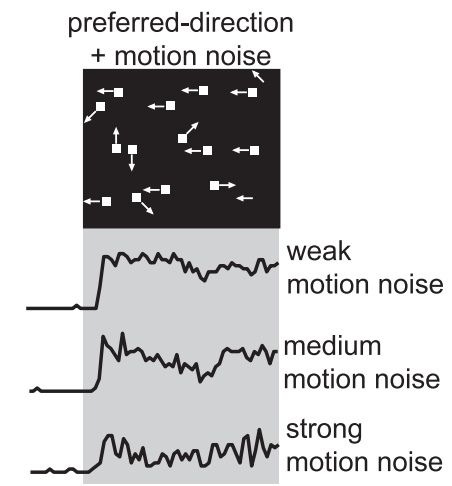

B

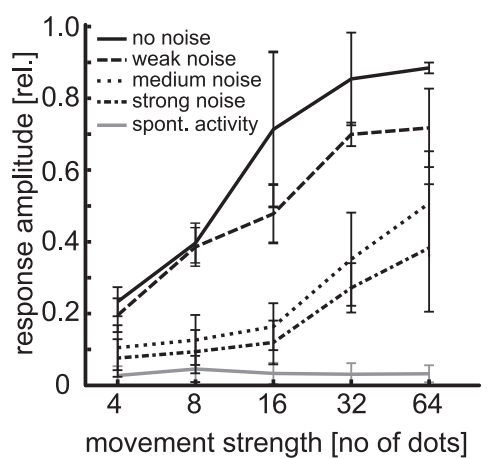

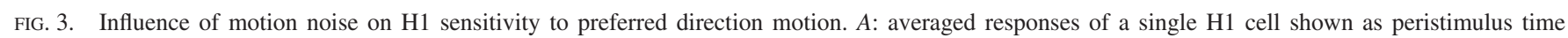

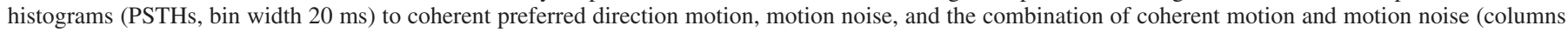

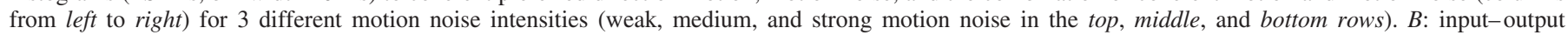

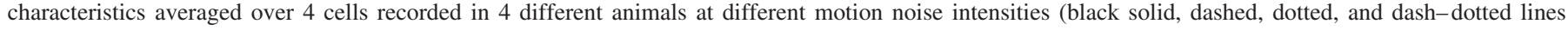
represent no, weak, medium, and strong motion noise, respectively; the gray solid line is the spontaneous activity). Error bars are SEs. 
quences on the response amplitude and the cell's sensitivity for preferred direction motion.

\section{Robust direction tuning}

In which way does motion noise affect the coding properties of blowfly motion-sensitive TCs? We used the motion noise to target the robustness of direction tuning of TCs. For this analysis $\mathrm{H} 1$ responses were recorded extracellularly to obtain sufficiently long and stable recordings. The coherent motion pattern moved in different directions ( $45^{\circ}$ steps) and was combined with motion noise. We used three conditions: 1) no motion noise, 2) medium, and 3) strong motion noise. The solid lines in Fig. 4 show the direction-tuning curves for coherent motion alone (64 or 16 motion dots in Fig. 4, $A$ and $B$, respectively) averaged across six $\mathrm{H} 1$ recordings. The response amplitude was normalized to the average response amplitude determined in the first reference section at the beginning of the trial where all 64 dots moved coherently in the cell's preferred direction (see METHODS). H1 exhibits the typical broad direction tuning, with an abnormal "dent" in the response amplitude at the "preferred direction." This seemingly surprising finding is in line with the conclusions drawn by Buchner (1976). Given the hexagonal lattice of the fly's eye, there are three ways to detect for example horizontal movement involving nearest-neighbor interactions (Fig. 4, inset, shown for left to right movement). In addition to interactions along the horizontal axis of the eye, there are also strong interactions along the oblique axes of the ommatidial lattice (Buchner 1976; Schuling et al. 1989). If the direction sensitivity of TCs is probed with grating patterns (Hausen 1976, 1982b) the dent in the tuning curve did not appear. In contrast to stripe patterns, which simultaneously activate all three types of movement detectors when moving horizontally, the dots used here (each dot's size approximates the acceptance angle of a single photoreceptor) stimulate only one type of detector at a time. Depending on the relative weight of the different types of nearest-neighbor interactions, dot stimuli may thus lead to the dent in the tuning curve observed here.

When the motion stimulus is combined with motion noise, the peak amplitudes of the direction tuning curve decrease dramatically (dashed curves in Fig. 4). The tuning width does
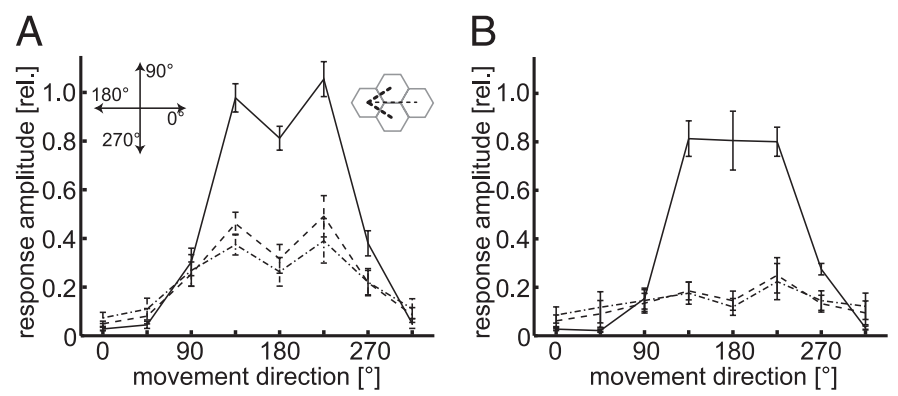

FIG. 4. H1 direction tuning when combined with motion noise. A: H1 responses as a function of the coherent motion direction (64 dots) without motion noise (solid line) and combined with medium and strong motion noise (dashed and dash-dotted lines, respectively); $180^{\circ}$ corresponds to the right $\mathrm{H} 1$ 's preferred direction. Plots are averages across $6 \mathrm{H} 1$ recordings done in 6 different animals. Error bars represent the SE. Right inset: ommatidial lattice of the fly compound eye. Dashed lines indicate the 3 different connections responding to horizontal motion from left to right. B: same as $A$ but with 16 dots moving coherently. not significantly change for the combinations of coherent motion with medium and strong motion noises (Fig. 4, dashed and dash-dotted lines). Rather than sharpening, the tuning curves become slightly broader. With a very weak motion stimulus of only 16 dots moving in different directions (Fig. $4 B)$, the motion noise reduces the response so much that the cell's direction tuning almost vanishes. Nevertheless, if we take into account that the very weak motion stimulus consists of only 16 dots and is combined with motion noise consisting of 112 dots moving in random directions, the direction tuning can be concluded to be quite robust against motion noise.

\section{Robust representation of pattern velocity}

From analyses with white noise velocity fluctuations it is known that the spike rate of the $\mathrm{H} 1$ cell represents the time course of velocity of a moving pattern up to frequencies of about $10 \mathrm{~Hz}$, as long as the velocities are sufficiently small (Bialek et al. 1991; Haag and Borst 1998; Warzecha et al. 1998). Here we tested the velocity coding of the H1 cell when the dynamic motion signal is corrupted by motion noise. The motion dots moved with a dynamic velocity profile, which contained frequencies $\leq 10 \mathrm{~Hz}$ (Fig. 5, left column). Motion noise of different strengths was added. To quantify the coding performance we calculated the coherence (Haag and Borst 1998; van Hateren and Snippe 2001) between the time-dependent velocity profile of the motion stimulus and the neuronal responses under three conditions: 1) without motion noise, 2) with weak motion noise, and 3) with strong motion noise (Fig. 5 , top, middle, and bottom plots). The time course and amplitude of the $\mathrm{H} 1$ responses to the first and the second conditions are very similar. The spike rate reflects the pattern velocity in the preferred direction to a certain extent, whereas the nulldirection velocity cannot be resolved because of the rectifying effect of spike generation. Like the response amplitude, the overall response power and the coherence spectra under these two conditions are very similar. The coherence is close to 0.9 for almost the entire tested frequency range (gray solid lines in Fig. 5). A noise-free linear system would give a coherence of one. The difference between one and the measured coherence may be attributable to both the noise and the nonlinearities in the fly visual motion pathway. To obtain an estimate of the relative contribution of these two sources, the expected coherences were calculated. The expected coherence (black solid lines in Fig. 5) is defined as the coherence between the individual responses and the mean response, which can be considered the noise-free response if a sufficiently large number of trials is averaged. The deviation of the expected coherence from one arises from noise in the responses (van Hateren and Snippe 2001). Consequently, the difference between measured and expected coherence reflects the nonlinearity of the system. The data shown in Fig. 5 are based on a single cell with 80 trials per condition. Neither the measured coherence nor the expected coherence changed much when fewer trials were evaluated (not shown). Thus the responses to dynamically varied pattern velocity are quite reliable and the $\mathrm{H} 1$ cell is well able to code stimulus velocity even in the presence of the motion noise.

When the strong motion noise is added the response amplitude drops in accordance with the results obtained with constant velocity motion. Nonetheless, the time course of the 
dynamic-velocity stimulus H1 responses
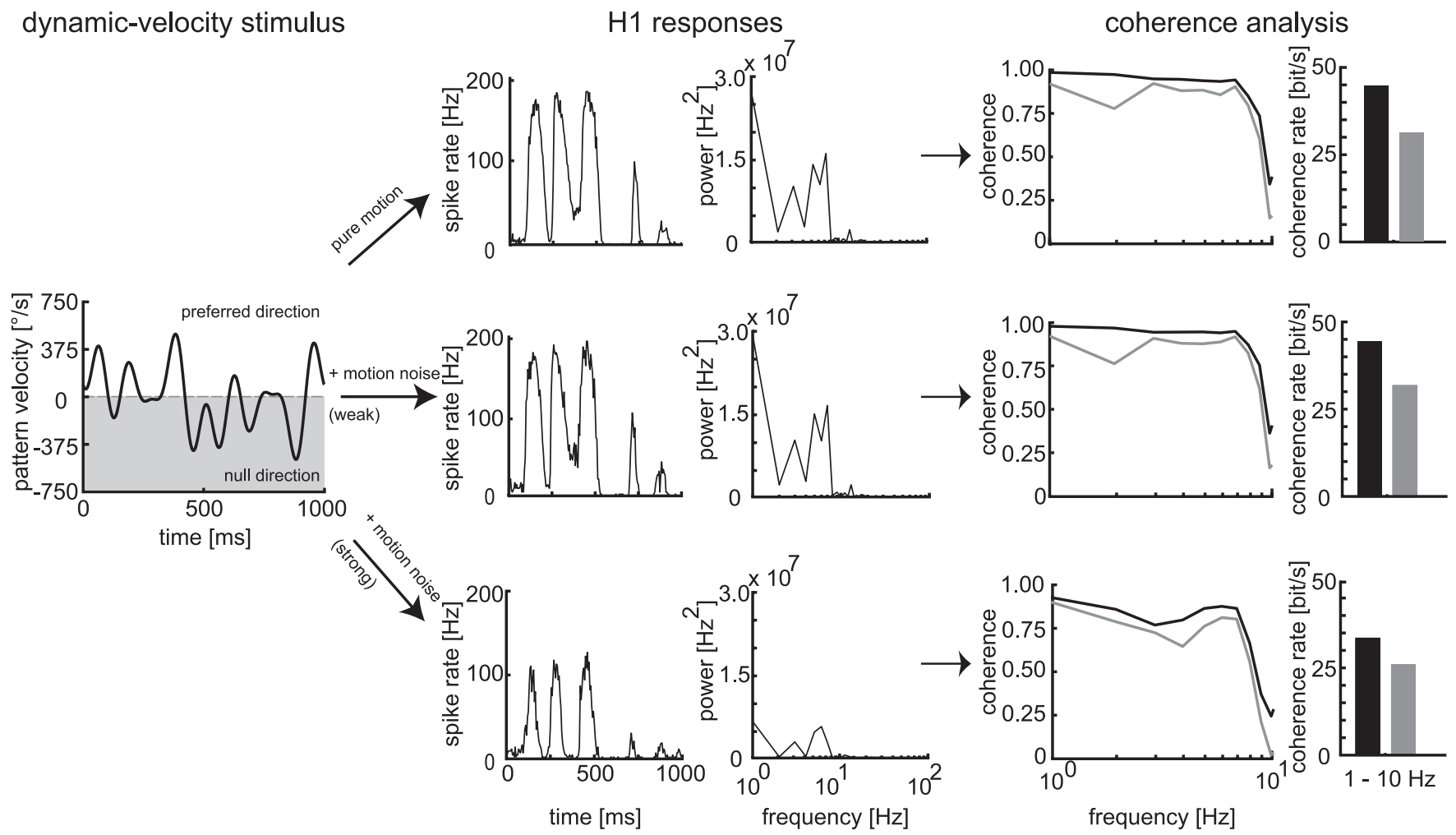

FIG. 5. Representation of pattern velocity in the H1 responses. Left column: stimulus time course. Positive and negative velocities denote movement in the preferred and null directions, respectively. Coherent motion following this velocity profile was presented without motion noise and combined with weak and strong motion noise. Middle column: averaged responses of a single H1 cell (80 stimulus presentations per condition) as PSTHs and the response power spectrums. Right column: coherence (gray solid line) and expected coherence (black solid line) spectra and expected coherence rates (black) and the coherence rates (gray) obtained under the 3 conditions (no, weak, and strong motion noise, top to bottom).

response is still very similar to that obtained with weak or without noise (compare the reduction in Figs. 3 and 4). With the reduced response amplitude the response power drops as well but the coherence declines only slightly. Although the decrease is much smaller than the corresponding reduction in spike frequency, the coherence remains high (between 0.6 and 0.8 ). This reflects that the time course of the $\mathrm{H} 1$ response scarcely changes and that the pattern velocity is still encoded very well even though motion noise reduces the spike rate to roughly 50\%. The expected coherence is somewhat lower for strong motion noise than under the other two stimulus conditions, suggesting that the noise in the responses increases when the strong motion noise is added to the stimulus.

The information transmitted by spike responses can be estimated from the coherence spectrum under certain conditions. For additive Gaussian noise, the so-called coherence rate is related to the Shannon information (van Hateren and Snippe 2001) and is given in bits per second. Even if these requirements are not met, the coherence rate can still be treated as an approximation of the Shannon information. Figure 5 (rightmost plots) shows the coherence rates and the expected coherence rates for the three conditions. For this sample recording the coherence and expected coherence rates are about the same for the conditions with and without weak motion noise (31.6 and $30.4 \mathrm{bits} / \mathrm{s}$ coherence rates and 41.3 and $43.5 \mathrm{bits} / \mathrm{s}$ expected coherence rates), but are reduced to a coherence rate of 26.3 $\mathrm{bits} / \mathrm{s}$ and an expected coherence rate of $33.7 \mathrm{bits} / \mathrm{s}$ when strong motion noise is added. Again, the coherence rate in the presence of strong motion noise still amounts to $86.5 \%$ of the coherence rate obtained under the no motion noise condition, although the spike rate dropped to only $43.6 \%(49.5 \pm 3.25$ and $21.6 \pm 2.5$ spikes, for no noise and strong motion noise, respectively). Thus the contribution of a single spike to the coherence rate is higher when strong motion noise is added to the dynamic motion stimulus than without added noise. Figure 6 shows the coherence rate per spike for the two conditions (black symbols) as an average over five cells. We find the coherence rate per spike almost doubled $(0.55 \pm 0.06$ to $1.07 \pm 0.22 \mathrm{bit} / \mathrm{spike}$ under the no-noise condition and strong motion noise condition, respectively). Similar results were

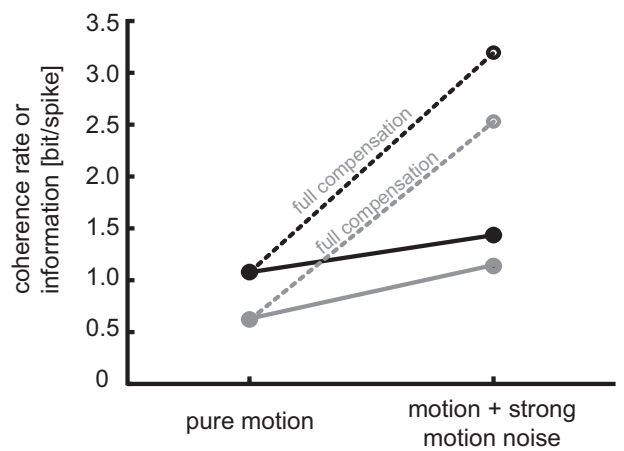

FIG. 6. Information content of individual spikes. Information transmitted by each single spike is shown for 2 conditions (no motion noise and combined with strong motion noise). Information was estimated from the coherence analysis (black symbols) and according to Brenner et al. (2001) (gray symbols). Open symbols represent the information content that would be needed to fully compensate the loss of spikes. 
obtained using a different measure (Brenner et al. 2000), which estimates the information transmitted per spike on the basis of the time-dependent spike rate $(0.912 \pm 0.10$ and $1.22 \pm 0.26$ bit/spike for the two conditions; gray symbols in Fig. 6). Despite the increased information per spike the total transmitted information decreases. The dashed lines in Fig. 6 indicate the coherence rate (information) per spike that would be needed to fully counterbalance the decreased spike rate when strong noise is added to the dynamic motion stimulus. A similar change in the information per spike was found in the context of motion adaptation: the loss of spikes was largely balanced by an increased information per spike (Heitwerth et al. 2005).

To conclude, $\mathrm{H} 1$ is well able to encode the time course of pattern velocity, even if a dynamically moving pattern is combined with strong motion noise that reduces the response amplitude quite drastically. The loss of spikes is partly compensated by the increased information carried by individual spikes.

\section{I S C US S I O N}

We addressed how different types of synaptic inputs interact on dendritic trees under in vivo conditions. By manipulating the natural sensory input of fly tangential cells (TCs), we find that visually induced responses and the input resistance of the cells are strongly affected by motion noise that was designed to be balanced and not to drive the cell on its own. Although the response amplitudes to coherent visual motion are strongly reduced in the presence of motion noise, functionally important properties such as the direction tuning and the ability to encode time-varying motion are robust against motion noise. From these results we hypothesize that the integration of functionally different synaptic inputs is a mechanism that inherently equalizes the ensemble's sensitivity irrespective of the specific stimulus conditions.

\section{Integration of functionally different synaptic inputs}

Inputs to neurons in the primate lateral geniculate nucleus were classified as drivers or modulators depending on their origin (Sherman and Guillery 1998). For rat cortical neurons a classification was suggested based on the context in which the inputs are activated rather than on their origin (Chance et al. 2002). Those excitatory and inhibitory inputs that are active at different times drive the neuron, whereas those active simultaneously cancel each other and constitute the modulating input. Simultaneous activation of excitatory and inhibitory inputs affects the membrane potential or the firing rate only slightly, but increases the membrane conductance and therefore represents a shunting input. In our stimulus design the impact of a dot of the motion noise moving in one direction is counteracted by another dot moving in the opposite direction. Thus the motion noise as a whole constitutes a modulating input and does not drive the cell on its own. In contrast, those inputs that are activated by coherent motion drive the neuron by either depolarizing or hyperpolarizing it, depending on the direction of motion. Motion noise was found to reduce the cell's sensitivity to simultaneously presented coherent motion by reducing the cell's input resistance and can therefore be considered as a shunting input to the cell. Even though this classification of the synaptic inputs into drivers and modulators is artificial in the case of TCs, especially because both components are embedded in the same visual input, it is useful for the system analysis presented here. In the real in vivo situation the actual role of an input signal depends on the context in which it is active, as was proposed for cortical neurons (Chance et al. 2002).

The specific consequences of a shunting input on dendritic computation of driving inputs is still controversially discussed. On the level of the postsynaptic potential, a decreased input resistance acts divisively on driving inputs (e.g., Holt and Koch 1997). The slope of the input-output characteristic is reduced as the membrane conductance increases. Things appear differently when the postsynaptic potential is converted into spikes. Several in vitro and modeling studies concluded that a tonic shunt then shifts the input-output characteristic along the input axis (Berman et al. 1992; Connors et al. 1988; Holt and Koch 1997). This is often described as a subtractive effect because it resembles the effect of a hyperpolarizing input, although the underlying postsynaptic potential is in fact divisively modulated (e.g., Holt and Koch 1997). Recent analyses mimicking the natural synaptic input by the dynamic-clamp technique concluded that shunting can modulate a neuron's gain resulting in slope changes of the input-output characteristic (Chance et al. 2002; Fellous et al. 2003; Mitchell and Silver 2003). Prerequisite is an increased membrane potential noise induced as a by-product of the shunting input as is characteristic of synaptic signals. Consequently, driving stimuli, which on their own would not suffice to induce spiking, are enabled to cross the spike threshold, an effect reminiscent of stochastic resonance (e.g., Wiesenfeld and Moss 1995).

In our experiments done under in vivo conditions, the shunting effect and the increased membrane potential fluctuations induced by the modulating input (Fig. 1) lead, at least in principle, to conditions for a divisive change of the spike response. However, the changes observed in the input-output characteristics (Figs. 2 and 3) do not allow us to infer a shift or a slope change as the exclusive mechanism. On the other hand, subtractive and divisive mechanisms affect tuning curves of spiking neurons very differently: in the case of a subtractive shift, nonoptimal directions fail to induce spiking and the tuning curve is sharpened - the so-called iceberg effect (e.g., Anderson et al. 2000). As a consequence of a divisive mechanism the overall spike rate is scaled but the width of the tuning curve stays the same. Thus the observed reduction in directional gain with an almost unaffected tuning width (Fig. 4) suggests a divisive in favor of a subtractive effect on the H1 spike responses. As mentioned above, this might be the consequence of the increased membrane potential fluctuations at higher motion noise levels, which allow occasional action potential generation even at suboptimal directions, i.e., when the average postsynaptic potential is below spike threshold. This is in line with conclusions drawn by Anderson et al. (2000), who showed that membrane potential noise is the key for contrast-invariant tuning curves in cat complex and simple cells.

\section{Alternative explanations of gain control}

Similar changes of the input-output characteristics as described above are also found in simple cells of the macaque 
visual cortex. For instance, the contrast dependency of the response to an optimally oriented drifting grating is shifted when simultaneously presented with an orthogonal grating that has no driving impact of its own. Likewise, superimposed visual noise reduces the response to the drifting grating (Carandini and Heeger 1994; Carandini et al. 1997). These gain changes were interpreted by shunting input to the considered neuron mediated by another cortical neuron pooling many local retinotopic inputs (Carandini and Heeger 1994; Carandini et al. 1997). This scheme is reminiscent of a model originally proposed to account for gain control of fly TCs, which also relies on a shunting pool cell (Reichardt et al. 1983). Because in later studies this gain control could be explained in a more parsimonious way by realizing - in line with our present findings that the driving input not only drives the cell, but also leads to pronounced shunting (Borst et al. 1995) - a similar influence of the direct peripheral input of cortical cells on the cell's input resistance may also play a role in controlling the gain of these neurons.

\section{Robustness of temporal encoding properties}

Both effects of motion noise, the reduced gain and the increased membrane potential noise, could impair the coding performance of TCs. Surprisingly, the stimulus time course can be recovered from the neuronal responses almost as well in the presence of motion noise as in its absence, although the corresponding spike rate decreased to 50\%. This result seems to contradict findings that visual flicker impairs the ability of monkey area MT/MST neurons to discriminate between motion in different directions (Churan and Ilg 2002). This discrepancy may be a consequence of the method used to discriminate between neuronal responses. Churan and Ilg (2002) used a measure derived from signal detection theory to discriminate between two alternative directions of motion on the basis of the mean response to constant velocity stimuli combined with flicker. In contrast, we tested how well the time course of the dynamic stimulus can be recovered from the neuronal response.

\section{Functional implications}

The fly's visual system offers the opportunity to link neuronal performance to the animal's behavior (for reviews see Egelhaaf and Borst 1993; Egelhaaf et al. 2002; Hausen 1984). TCs are involved in the evaluation of optic flow and have been shown to encode information about the three-dimensional layout of the environment (Kern et al. 2005) as well as certain self-motions such as body roll (Karmeier et al. 2005; Krapp et al. 1998, 2001). Some TCs project directly to motoneurons that control compensatory head movements (Gilbert et al. 1995; Gronenberg et al. 1995; Milde et al. 1995; Strausfeld et al. 1987).

During aerobatic flight maneuvers the fly induces optic flow, which is characterized by peculiar patterns of motion vectors depending on the type of self-motion and the environmental layout. Although some maneuvers induce motion vectors optimally driving a certain TC, they are ineffective for other TCs (Karmeier et al. 2006). Irrespective of the efficiency of driving a specific combination of TCs at a given time, the input resistance and thus the gain of all TCs is decreased regardless of the actual stimulus situation. Thus under natural conditions the gains of all TCs can be expected to be about the same level at any time. This level may be low when the animal is flying rapidly and thus confronted mainly with rapid retinal motion, somewhat higher when the animal is walking and thus experiencing mainly slower retinal motion, and at its highest level when the animal is just sitting and thus experiencing virtually no motion at all. Thus all TCs are proposed to operate with a similar gain and thus a similar sensitivity to motion, irrespective of the overall strength of the optic flow and thereby the behavioral context. Thus the continuous bombardment with motion stimuli, although not necessarily driving all TCs, can equalize the network's gain, leaving it well able to code for different parameters as we could show for the direction tuning of constant motion or a dynamically varying stimulus.

\section{A C K N O W LED G M E N T S}

We are grateful to R. Kurtz and D. Brooks for critically reading the manuscript. We also thank two anonymous referees for helpful comments.

\section{G R A N T S}

This work was supported by a Volkswagen-Stiftung grant to A.-K. Warzecha.

\section{REFERENCES}

Anderson JS, Lampl I, Gillespie DC, and Ferster D. The contribution of noise to contrast invariance of orientation tuning in cat visual cortex. Science 290: 1968-1972, 2000.

Azouz R and Gray CM. Cellular mechanisms contributing to response variability of cortical neurons in vivo. J Neurosci 19: 2209-2223, 1999.

Berman NJ, Douglas RJ, and Martin KA. GABA-mediated inhibition in the neural networks of visual cortex. Prog Brain Res 90: 443-476, 1992.

Bialek W, Rieke F, de Ruyter van Steveninck RR, and Warland D. Reading a neural code. Science 252: 1854-1857, 1991.

Borst A, Egelhaaf M, and Haag J. Mechanisms of dendritic integration underlying gain control in fly motion-sensitive interneurons. J Comput Neurosci 2: 5-18, 1995.

Borst A and Haag J. The intrinsic electrophysiological characteristics of fly lobula plate tangential cells. I. Passive membrane properties. J Comput Neurosci 3: 313-336, 1996.

Borst A and Haag J. Neural networks in the cockpit of the fly. J Comp Physiol A Sens Neural Behav Physiol 188: 419-437, 2002.

Brenner N, Strong SP, Koberle R, Bialek W, and de Ruyter van Steveninck RR. Synergy in a neural code. Neural Comput 12: 1531-1552, 2000.

Brotz TM, Gundelfinger ED, and Borst A. Cholinergic and GABAergic receptors on fly tangential cells and their role in visual motion detection. J Neurophysiol 76: 1786-1799, 1996.

Buchner E. Elementary movement detectors in an insect visual system. Biol Cybern 24: 85-101, 1976.

Carandini M and Heeger DJ. Summation and division by neurons in primate visual cortex. Science 264: 1333-1336, 1994.

Carandini M, Heeger DJ, and Movshon JA. Linearity and normalization in simple cells of the macaque primary visual cortex. J Neurosci 17: 86218644, 1997.

Chance FS, Abbott LF, and Reyes AD. Gain modulation from background synaptic input. Neuron 35: 773-782, 2002.

Churan J and Ilg UJ. Flicker in the visual background impairs the ability to process a moving visual stimulus. Eur J Neurosci 16: 1151-1162, 2002.

Connors BW, Malenka RC, and Silva LR. Two inhibitory postsynaptic potentials, and GABAA and GABAB receptor-mediated responses in neocortex of rat and cat. J Physiol 406: 443-468, 1988.

Destexhe A, Rudolph M, and Paré D. The high-conductance state of neocortical neurons in vivo. Nat Rev Neurosci 4: 739-751, 2003.

Egelhaaf $M$ and Borst A. Motion computation and visual orientation in flies. Comp Biochem Physiol 104A: 659-673, 1993.

Egelhaaf M, Grewe J, Karmeier K, Kern R, Kurtz R, and Warzecha A-K. Novel approaches to visual processing in insects: case studies on neuronal 
computations in the blowfly. In: Methods in Insect Sensory Neuroscience, edited by Christensen TA. Boca Raton, FL: CRC Press, 2005, p. 185-212.

Egelhaaf M, Kern R, Krapp HG, Kretzberg J, Kurtz R, and Warzecha A-K. Neural encoding of behaviourally relevant visual-motion information in the fly. Trends Neurosci 25: 96-102, 2002.

Egelhaaf $\mathbf{M}$ and Warzecha A-K. Encoding of motion in real time by the fly visual system. Curr Opin Neurobiol 9: 454-460, 1999.

Fellous JM, Rudolph M, Destexhe A, and Sejnowski TJ. Synaptic background noise controls the input/output characteristics of single cells in an in vitro model of in vivo activity. Neuroscience 122: 811-829, 2003.

Franceschini N. Sampling of the visual environment by the compound eye of the fly: fundamentals and applications. In: Photoreceptor Optics, edited by Snyder AW and Menzel R. New York: Springer-Verlag, 1975, p. 98-125.

Gilbert C, Gronenberg W, and Strausfeld NJ. Oculomotor control in calliphorid flies: head movements during activation and inhibitions of neck motor neurons corroborate neuroanatomical predictions. J Comp Neurol 361: 285-297, 1995.

Gronenberg W, Milde JJ, and Strausfeld NJ. Oculomotor control in calliphorid flies: organization of descending neurons to neck motor neurons responding to visual stimuli. J Comp Neurol 361: 267-284, 1995.

Haag J and Borst A. Active membrane properties and signal encoding in graded potential neurons. J Neurosci 18: 7972-7986, 1998.

Hausen K. Functional characterization and anatomical identification of motion sensitive neurons in the lobula plate of the blowfly Calliphora erythrocephala. Z Naturforsch 31c: 629-633, 1976.

Hausen K. Motion sensitive interneurons in the optomotor system of the fly. I. The horizontal cells: structure and signals. Biol Cybern 45: 143-156, 1982 a.

Hausen K. Motion sensitive interneurons in the optomotor system of the fly. II. The horizontal cells: receptive field organization and response characteristics. Biol Cybern 46: 67-79, 1982b.

Hausen K. The lobula-complex of the fly: structure, function and significance in visual behaviour. In: Photoreception and Vision in Invertebrates, edited by Ali MA. New York: Plenum Press, 1984, p. 523-559.

Heitwerth J, Kern R, van Hateren JH, and Egelhaaf M. Motion adaptation leads to parsimonious encoding of natural optic flow by blowfly motion vision system. J Neurophysiol 94: 1761-1769, 2005.

Hengstenberg R. Common visual response properties of giant vertical cells in the lobula plate of the blowfly Calliphora. J Comp Physiol 149: 179-193, 1982.

Holt GR and Koch C. Shunting inhibition does not have a divisive effect on firing rates. Neural Comput 9: 1001-1013, 1997.

Karmeier K, Krapp HG, and Egelhaaf M. Population coding of self-motion: applying Bayesian analysis to a population of visual interneurons in the fly. J Neurophysiol 94: 2182-2194, 2005.

Karmeier K, van Hateren JH, Kern R, and Egelhaaf M. Encoding of naturalistic optic flow by a population of blowfly motion sensitive neurons. J Neurophysiol 96: 1602-1614, 2006

Kern R, van Hateren JH, Michaelis C, Lindemann JP, and Egelhaaf M. Function of a fly motion-sensitive neuron matches eye movements during free flight. PLoS Biol 3: e171, 2005.

Koch C, Poggio T, and Torre V. Nonlinear interactions in a dendritic tree: localization, timing, and role in information processing. Proc Natl Acad Sci USA 80: 2799-2802, 1983.
Krapp HG, Hengstenberg B, and Hengstenberg R. Dendritic structure and receptive-field organization of optic flow processing interneurons in the fly. J Neurophysiol 79: 1902-1917, 1998.

Krapp HG, Hengstenberg R, and Egelhaaf M. Binocular contributions to optic flow processing in the fly visual system. J Neurophysiol 85: 724-734, 2001.

Kretzberg J, Egelhaaf M, and Warzecha AK. Membrane potential fluctuations determine the precision of spike timing and synchronous activity: a model study. J Comput Neurosci 10: 79-97, 2001.

Milde JJ, Gronenberg W, and Strausfeld NJ. Oculomotor control in calliphorid flies: organization of descending neurons to neck motor neurons responding to visual stimuli. J Comp Neurol 361: 267-284, 1995.

Mitchell SJ and Silver RA. Shunting inhibition modulates neuronal gain during synaptic excitation. Neuron 38: 433-445, 2003.

Prescott SA and De Koninck Y. Gain control of firing rate by shunting inhibition: roles of synaptic noise and dendritic saturation. Proc Natl Acad Sci USA 100: 2076-2081, 2003.

Prinz AA, Abbott LF, and Marder E. The dynamic clamp comes of age. Trends Neurosci 27: 218-224, 2004.

Reichardt W, Poggio T, and Hausen K. Figure-ground discrimination by relative movement in the visual system of the fly. Part II. Towards the neural circuitry. Biol Cybern Suppl 46: 1-30, 1983.

Schuling FH, Mastebroek HAK, Bult R, and Lenting BPM. Properties of elementary movement detectors in the fly Calliphora erythrocephala. J Comp Physiol A Sens Neural Behav Physiol 165: 179-192, 1989.

Sherman SM and Guillery RW. On the actions that one nerve cell can have on another: distinguishing "drivers" from "modulators." Proc Natl Acad Sci USA 95: 7121-7126, 1998.

Single S, Haag J, and Borst A. Dendritic computation of direction selectivity and gain control in visual interneurons. J Neurosci 17: 6023-6030, 1997.

Strausfeld NJ, Seyan HS, and Milde JJ. The neck motor system of the fly Calliphora erythrocephala. I. Muscles and motor neurons. J Comp Physiol A Sens Neural Behav Physiol 160: 205-224, 1987.

van Hateren JH, Kern R, Schwertfeger G, and Egelhaaf M. Function and coding in the blowfly $\mathrm{H} 1$ neuron during naturalistic optic flow. J Neurosci 25: 4343-4352, 2005.

van Hateren JH and Snippe HP. Information theoretical evaluation of parametric models of gain control in blowfly photoreceptor cells. Vision Res 41: 1851-1865, 2001.

Warzecha AK, Egelhaaf M, and Borst A. Neural circuit tuning fly visual interneurons to motion of small objects. I. Dissection of the circuit by pharmacological and photoinactivation techniques. J Neurophysiol 69: 329339, 1993.

Warzecha AK, Kretzberg J, and Egelhaaf M. Temporal precision of the encoding of motion information by visual interneurons. Curr Biol 8: 359368, 1998.

Warzecha AK, Kretzberg J, and Egelhaaf M. Reliability of a fly motionsensitive neuron depends on stimulus parameters. J Neurosci 20: $8886-$ 8896, 2000.

Wiesenfeld K and Moss F. Stochastic resonance and the benefits of noise: from ice ages to crayfish and squids. Nature 373: 33-36, 1995. 